\title{
Optimal Finite-Time State Estimation for Discrete-Time Switched Systems under Switching Frequency Constraint
}

\author{
Lin Du, ${ }^{1}$ Weiming Xiang, ${ }^{1}$ and Yongchi Zhao ${ }^{2}$ \\ ${ }^{1}$ School of Transportation and Logistics, Southwest Jiaotong University, Chengdu, Sichuan 610031, China \\ ${ }^{2}$ School of Electrical Engineering, Southwest Jiaotong University, Chengdu, Sichuan 610031, China \\ Correspondence should be addressed to Lin Du; dlswjtu@163.com
}

Received 16 April 2014; Revised 19 June 2014; Accepted 4 July 2014; Published 24 July 2014

Academic Editor: Xi-Ming Sun

Copyright (c) 2014 Lin Du et al. This is an open access article distributed under the Creative Commons Attribution License, which permits unrestricted use, distribution, and reproduction in any medium, provided the original work is properly cited.

\begin{abstract}
The state estimation problem for a class of switched linear systems which only switches in some short interval is addressed. Besides the asymptotic stability of error dynamics, the boundness of error state is a significant issue for short-time switched systems. By introducing the concept of finite-time stability, the state estimation procedure is formulated to determine appropriate observer gains ensuring the error dynamics is finite-time stable in the short-time switching intervals of interest. Optimal finite-time observers are designed through iterative algorithms to minimize the bound of error state, in the cases with and without disturbances. Particularly, when the total activation time is known, a less conservative result can be derived and an optimization problem can be solved with the help of the genetic algorithm. A numerical example is provided to illustrate the theoretical findings in this paper.
\end{abstract}

\section{Introduction}

Switched systems, as typical hybrid dynamical system that is composed of a family of subsystems described by differential or difference equations and a switching rule orchestrating the switching between the subsystems, have attracted much attention in control theory and practice during recent decades. Switched systems can be efficiently used to model many practical systems which are inherently multimodel, in the sense that several dynamical systems are required to describe their behaviours. For example, many physical processes exhibit switched and hybrid nature [1-3], and switched systems arise in many engineering applications [48]. Generally, the stability and stabilization problems are the main concerns in the field of switched systems [9-13]. For more details of the recent results of the basic problems in stability and stabilization for switched systems, the reader is referred to the survey paper [14] and the references cited therein.

On the other hand, the issue of state estimation has been investigated intensively in continuous and discrete domain. After Luenberger proposed a method to design an observer for linear time invariant system in 1960s [15], numerous results on Luenberger-like observer design were developed. Recently, there are some results about observer design for switched systems and most of them are about switched linear systems. In [16, 17] a common quadratic Lyapunov function which guarantees the error system stability was used to design observer for switched continuous-time and discretetime linear systems and observer design for a class of switched nonlinear systems was investigated in [18]. Pettersson proposed an approach using multiple Lyapunov functions to design an observer for switched linear systems [19]. Full and reduced order observers for a class of linear switched control systems are studied in [20].

It is worth mentioning that most results about state estimation for switched systems only focus on asymptotic observer which is defined on the infinite time interval; few results concerned with the error dynamics performance in a finite-time interval have been reported, which is also interesting and important in both theory and actual applications. The boundness of state during a fixed interval has been studied extensively based on the notion called finite-time stability [21-23], and it has been extended to analyze state 
boundness of switched system recently [24-26]. But the boundness of error state in the state estimation problem has not been fully studied. It is well known that frequent switching between stable subsystems can lead to instability due to the obvious reason that the overshoot caused by the transient of subsystem response may destroy the stability [27]. The boundness of state during a fixed interval, which almost relies on the transient response, is naturally supposed to be significantly affected by switching among several subsystems. Therefore, based on the notion of switching frequency, which describes how frequently the switching occurs in a time interval, the optimal finite-time observer design problem is considered.

The main contribution in this paper lies in the optimal finite-time observer design. At first, without considering disturbance, the optimal finite-time observer is designed to minimize the bound of error state by an LMI based iterative algorithm; then, while the $\mathscr{H}_{\infty}$ performance is taken into account, an optimal observer is constructed to minimize error state bound in regard to an $\mathscr{H}_{\infty}$ performance. In both cases, the results are relevant to the switching frequency. Furthermore, if more information about switching signal is known, that is, the total activation time of each subsystem is available, a less conservative result can be achieved, and genetic algorithm is introduced in observer design procedure.

The rest of this paper is organized as follows. In Section 2 the problem formulation and some preliminaries are introduced; the main results on optimal observer design are proposed in Section 3. Some further discussion on improved results is given in Section 4. A numerical is provided in Section 5. Conclusions are given in Section 6.

\section{Preliminaries and Problem Formulation}

In this paper, a switched discrete-time system is considered as follows:

$$
\begin{aligned}
\Sigma: x(k+1) & =A_{\sigma(k)} x(k)+B_{\sigma(k)} u(k)+E_{\sigma(k)} \omega(k), \\
y(k) & =C_{\sigma(k)} x(k)+D_{\sigma(k)} u(k)+F_{\sigma(k)} \omega(k),
\end{aligned}
$$

where $x(k) \in \mathbf{R}^{n}$ is the discrete-time state, $u(k) \in \mathbf{R}^{m}$, $\omega(k) \in \mathbf{R}^{p}$, and $y(k) \in \mathbf{R}^{q}$ are the input vector, disturbance, and measured output. $\sigma(k):[0, \infty) \rightarrow \mathscr{I}:=\{1,2, \ldots, N\}$ is a piecewise constant function of time, called switching law or switching signal, which takes value in a finite index set $\mathscr{I}:=\{1,2, \ldots, N\} . N>0$ is the number of subsystems. $A_{i}$, $B_{i}, C_{i}, D_{i}, E_{i}$, and $F_{i}$ are constant matrices with appropriate dimensions.

To describe the short-time switching property considered in this paper, we introduce the time interval sequence denoted by $\overline{\mathcal{S}}:=\left\{\Gamma_{1}^{s}, \Gamma_{1}^{l}, \Gamma_{2}^{s}, \Gamma_{2}^{l}, \ldots, \Gamma_{n}^{s}, \Gamma_{n}^{l}, \ldots\right\}$, where $\Gamma_{n}^{s}:=$ $\left[t_{n, 0}^{s}, t_{n, 0}^{s}+T_{n}^{s}\right)$ represents the so-called short-time switching interval in which switching occurs and $\Gamma_{n}^{l}:=\left[t_{n, 0}^{l}, t_{n, 0}^{l}+T_{n}^{l}\right)$ denotes the relatively long interval in which the system is maintained in a fixed mode $i$. Since the system exhibits the short-time switching property, the following assumption is proposed:
Assumption 1. Consider the time interval sequence $\bar{\delta}$; it is assumed that for all $n$, the following two conditions are satisfied: (1) $T_{n}^{l} \gg T_{n}^{s}$; (2) $t_{n, 0}^{s}+T_{n}^{s}=t_{n, 0}^{l}$ and $t_{n, 0}^{l}+T_{n}^{l}=t_{n+1,0}^{s}$.

Remark 2. These two assumptions are necessary for time interval sequence $\bar{\delta}$ to describe the short-time switching property. The first condition in Assumption 1 implies that the switching occurs in some short-time interval and in most of the other time no switching exists. Furthermore, since $T_{n}^{l} \gg$ $T_{n}^{s}$, it indicates that the relatively long intervals are sufficiently long so that the stability is explicitly dependent on the stability property of subsystem $i$ activated in relatively long interval $\Gamma_{n}^{l}$. Then, the second condition makes sure the sequence $\overline{\mathcal{S}}$ is well-posed defined in $[0, \infty)$; that is, $\bigcup_{n=1,2, \ldots}\left(\Gamma_{n}^{s} \cup \Gamma_{n}^{l}\right)=[0, \infty)$ and $\Gamma_{n}^{s} \cap \Gamma_{m}^{l}=\emptyset$, for all $n, m$.

In the interval $\Gamma_{n}^{s}$, the switching sequence can be defined as $\delta_{n}:=\left\{k_{0}^{n}, k_{1}^{n}, \ldots, k_{m}^{n}, \ldots, k_{M}^{n}\right\}$, where $k_{0}^{n}$ denotes the initial instant of $\Gamma_{n}^{s}, k_{m}^{n}$ denotes the $m$ th switching instant in $\Gamma_{n}^{s}$, and $k_{M}^{n}$ stands for the last instant in $\Gamma_{n}^{s}$, respectively. Explicitly, the length of interval $\Gamma_{n}^{s}$ can be figured out as $\mathscr{T}_{n}=k_{M}^{n}-k_{0}^{n}$.

Definition 3. For each switching signal $\sigma(k)$ and short-time interval $\Gamma_{n}^{s}$, the switching frequency $f_{n}$ is defined by $f_{n}=$ $M /\left(k_{M}^{n}-k_{0}^{n}\right)=M / \mathscr{T}_{n}$.

Assumption 4. The switching between system and observer is synchronous; that is, the activated subsystem is explicitly known at each switching instant and the designed corresponding observer can be activated immediately.

In this paper, we focus on the state estimation problem and consider a Luenberger-type observer $\Sigma_{o}$ of the following form:

$$
\begin{aligned}
\Sigma_{o}: \widehat{x}(k+1)= & A_{\sigma(k)} \widehat{x}(k)+B_{\sigma(k)} u(k) \\
& +L_{\sigma(k)}\left(y(k)-C_{\sigma(k)} \widehat{x}(k)-D_{\sigma(k)} u(k)\right),
\end{aligned}
$$

where $\hat{x} \in \mathbf{R}^{n}$ is the estimated state and matrices $L_{i} \in$ $\mathbf{R}^{n \times q}$ are the observer gains to be designed. Letting $e(k)=$ $x(k)-\widehat{x}(k)$, one can easily obtain the error dynamics $\Sigma_{e}$ under synchronous switching as follows:

$$
\begin{aligned}
\Sigma_{e}: e(k+1)= & \left(A_{\sigma(k)}-L_{\sigma(k)} C_{\sigma(k)}\right) e(k) \\
& +\left(E_{\sigma(k)}-L_{\sigma(k)} F_{\sigma(k)}\right) \omega(k) .
\end{aligned}
$$

As for switched system with short-time switching signal, the asymptotic stability of the error dynamics is equivalent to the asymptotic stability of each error subsystem; however, in actual applications, the asymptotic stability is not enough for short-time switched system since the frequent switching behaviours may cause the error states to reach a very large value, which is not acceptable in the state estimation process; for example, see examples in [24]. Hence, the concept of finite-time stability is introduced.

Definition 5. Switched error system (3) with $\omega(k) \equiv 0$ is said to be finite-time stable with respect to $(\delta, \varepsilon, R, M)$, where $0 \leq$ 
$\delta<\varepsilon, R$ is a positive definite matrix, and a scalar $M>0$, if $e^{T}(k) \operatorname{Re}(k)<\varepsilon^{2}$, for all $k \in\{1,2, \ldots, M\}$ whenever $e_{0}^{T} \operatorname{Re}_{0}<$ $\delta^{2}$.

As for the finite-time observer design problem which is supposed to guarantee the error state in a prescribed boundary, the bound $\varepsilon$ is required to be made as small as possible. Thus, the first optimal state observer design problem for a finite-time interval $\Gamma_{n}^{s}$ with $\mathscr{T}_{n}=k_{M}^{n}-k_{0}^{n}$ is given as follows.

Problem 6. Given switched system (1a) and (1b) with $\omega(k) \equiv$ 0 , design an optimal observer ensuring error dynamics (3) is finite-time stable with respect to $\left(\delta, \varepsilon, R, \mathscr{T}_{n}\right)$ and minimizing $\varepsilon$ as small as possible.

When the switched system is subjected to external input signals $\omega(k)$, which is assumed to be energy bounded in the finite-time interval $\left[k_{0}^{n}, k_{M}^{n}\right]$ that is, $\sum_{k=k_{0}^{n}}^{k_{M}^{n}} \omega^{T}(k) \omega(k)<d^{2}$, where $d \geq 0$, then, the finite-time boundedness and that with $\mathscr{H}_{\infty}$ performance are considered.

Definition 7. Switched error system (3) is said to be finite-time bounded with respect to $(\delta, \varepsilon, d, R, M)$, where $0 \leq \delta<\varepsilon, R$ is a positive definite matrix, and a scalar $M>0$, if $e^{T}(k) \operatorname{Re}(k)<$ $\varepsilon^{2}$, for all $k \in\{1,2, \ldots, M\}$ whenever $e_{0}^{T} \operatorname{Re}_{0}<\delta^{2}$.

Concerned with $\mathscr{H}_{\infty}$ performance problem, the controlled output for error state $e(t)$ is given as

$$
z(k)=H_{\sigma(k)} e(k)+G_{\sigma(k)} \omega(k),
$$

where $H_{i}, G_{i}, i \in \mathscr{I}$, are known matrices.

Definition 8. Switched error system (3)-(4) is said to be $\mathscr{H}_{\infty}$ finite-time boundness with respect to $(\delta, \varepsilon, d, \gamma, R, M)$, where $0 \leq \delta\langle\varepsilon, \gamma\rangle 0, R$ is a positive definite matrix, and a scalar $M>0$, if error system (3)-(4) is finite-time bounded with respect to $(\delta, \varepsilon, d, R, M)$ and under zero-initial condition the output $z(k)$ satisfies

$$
\sum_{k=0}^{M} z^{T}(k) z(k)<\gamma^{2} \sum_{k=0}^{M} \omega^{T}(k) \omega(k)
$$

When the $\mathscr{H}_{\infty}$ performance index $\gamma>0$ is taken into account, the minimum value of the state bound $\varepsilon$ is also of interest. Given a prescribed $\mathscr{H}_{\infty}$ performance, $\gamma>0$, the following problem is formulized to describe the optimal $\mathscr{H}_{\infty}$ finite-time observer design problem.

Problem 9. Given switched system (1a) and (1b), construct an optimal observer ensuring the $\mathscr{H}_{\infty}$ finite-time boundedness of error dynamics with respect to $\left(\delta, \varepsilon, d, \gamma, R, \mathscr{T}_{n}\right)$ and minimizing $\varepsilon$ as small as possible.

\section{Optimal Finite-Time Observer Design}

Before giving our results, some explicit facts are recalled. For a symmetric positive definite matrix $R \in \mathbf{R}^{n \times n}$, it is easy to verify that $R$ can be factorized according to $R=\left(R^{1 / 2}\right)^{T} R^{1 / 2}$, where $R^{1 / 2} \in \mathbf{R}^{n \times n}$ is a symmetric positive definite matrix. And for any positive definite matrix, $R \in \mathbf{R}^{n \times n}$, there always exists $R^{-1} \in \mathbf{R}^{n \times n}$ which is positive definite. At first, we consider Problem 6 where the disturbance $\omega(k) \equiv 0$; our first result is proposed as follows.

Theorem 10. Consider switched system (1a) and (1b) with $\omega(k) \equiv 0$ and switching frequency $f_{n}$. If there exist a set of matrices $P_{i}, X_{i}$ and scalars $\lambda_{1}>0, \lambda_{2}>0$, and $\eta \geq 1$ such that

$$
\begin{gathered}
\lambda_{1} R<P_{i}<\lambda_{2} R, \\
{\left[\begin{array}{cc}
-\eta P_{i} & A_{i}^{T} P_{i}-C_{i}^{T} X_{i}^{T} \\
P_{i} A_{i}-X_{i} C_{i} & -P_{i}
\end{array}\right]<0,} \\
\left(\frac{\lambda_{2}}{\lambda_{1}}\right)^{1+f_{n} \mathscr{T}_{n}} \delta^{2} \eta^{\mathscr{T}_{n}}-\varepsilon^{2}<0,
\end{gathered}
$$

then error dynamics (3) with $L_{i}=P_{i}^{-1} X_{i}$ is finite-time stable with respect to $\left(\delta, \varepsilon, R, \mathscr{T}_{n}\right)$.

Proof. Substituting $X_{i}=P_{i} L_{i}$ into (7), it follows that

$$
\left[\begin{array}{cc}
-\eta P_{i} & \left(A_{i}-L_{i} C_{i}\right)^{T} P_{i} \\
P_{i}\left(A_{i}-L_{i} C_{i}\right) & -\eta P_{i}
\end{array}\right]<0
$$

which implies $\left(A_{i}-L_{i} C_{i}\right)^{T} P_{i}\left(A_{i}-L_{i} C_{i}\right)-\eta P_{i}<0$.

Letting $V_{i}(k)=e^{T}(k) P_{i} e(k), i \in \mathscr{I}$ for each subsystem of error dynamics (3) with $\omega(k) \equiv 0$, we have

$$
V_{i}(k+1)<\eta V_{i}(k), \forall k \in \Gamma_{n}^{s} \backslash \mathcal{S}_{n} .
$$

Then, if the switched system switches from subsystem $i$ to $j$ at switching instant $k_{m}^{n} \in \mathcal{S}_{n}$, from (6), it is easy to see that

$$
\begin{aligned}
\frac{V_{j}\left(k_{m}^{n}\right)}{V_{i}\left(k_{m}^{n}\right)} & =\frac{e^{T}\left(k_{m}^{n}\right) P_{j} e\left(k_{m}^{n}\right)}{e^{T}\left(k_{m}^{n}\right) P_{i} e\left(k_{m}^{n}\right)}<\frac{\lambda_{2} e^{T}\left(k_{m}^{n}\right) \operatorname{Re}\left(k_{m}^{n}\right)}{\lambda_{1} x^{T}\left(k_{m}^{n}\right) \operatorname{Re}\left(k_{m}^{n}\right)} \\
& =\frac{\lambda_{2}}{\lambda_{1}}, \quad \forall k_{m}^{n} \in \mathcal{S}_{n} .
\end{aligned}
$$

We let

$$
V(k)=\sum_{i \in \mathscr{F}} \theta_{i}(k) V_{i}(k)=\sum_{i \in \mathscr{I}} e^{T}(k) \theta_{i}(k) P_{i} e(k),
$$

where $\theta_{i}(\cdot): \mathbb{Z}^{+} \rightarrow\{0,1\}$ and $\sum_{i \in \mathcal{F}} \theta_{i}(k)=1$ is the indication function indicating the activated subsystem of error dynamics. Thus, from (10) and (11) we can obtain

$$
V(k)<\left(\frac{\lambda_{2}}{\lambda_{1}}\right)^{f_{n} \mathscr{T}_{n}} \eta^{\mathscr{T}_{n}} V\left(k_{0}^{n}\right), \forall k \in \Gamma_{n}^{\mathcal{S}},
$$

for all $i \in \mathscr{I}$ and $\forall k \in \Gamma_{n}^{\mathcal{S}}$, we can obtain

$$
\begin{aligned}
V(k) & =e^{T}(k) P_{i} e(k)=e^{T}(k) R^{1 / 2} Q_{i} R^{1 / 2} e(k) \\
& \geq \inf _{i \in \mathscr{I}}\left\{\lambda_{\min }\left(Q_{i}\right)\right\} e^{T}(k) \operatorname{Re}(k),
\end{aligned}
$$

where $Q_{i}=R^{-1 / 2} P_{i} R^{-1 / 2}, i \in \mathscr{I}$. 
On the other hand, for all $i \in \mathscr{I}$, one sees

$$
\begin{aligned}
\eta^{k-k_{0}^{n}} V\left(k_{0}^{n}\right) & =\eta^{k-k_{0}^{n}} e^{T}\left(k_{0}^{n}\right) P_{i} e\left(k_{0}^{n}\right) \\
& =\eta^{k-k_{0}^{n}} e^{T}\left(k_{0}^{n}\right) R^{1 / 2} Q_{i} R^{1 / 2} e\left(k_{0}^{n}\right) \\
& \leq \eta^{k-k_{0}^{n}} \sup _{i \in \mathscr{I}}\left\{\lambda_{\max }\left(Q_{i}\right)\right\} e^{T}\left(k_{0}^{n}\right) \operatorname{Re}\left(k_{0}^{n}\right) .
\end{aligned}
$$

Using the fact that $\eta \geq 1$ and $e^{T}\left(k_{0}^{n}\right) \operatorname{Re}\left(k_{0}^{n}\right) \leq \delta^{2}$, we get

$$
\eta^{k-k_{0}^{n}} V\left(k_{0}^{n}\right) \leq \eta^{\mathscr{T}_{n}} \sup _{i \in \mathscr{I}}\left\{\lambda_{\max }\left(Q_{i}\right)\right\} \delta^{2}
$$

where $Q_{i}=R^{-1 / 2} P_{i} R^{-1 / 2}, i \in \mathscr{I}$. Altogether (13) (16), the following inequality can be derived:

$$
\begin{aligned}
& e^{T}(k) \operatorname{Re}(k) \\
& \quad<\left(\frac{\lambda_{2}}{\lambda_{1}}\right)^{f_{n} \mathscr{T}_{n}} \delta^{2} \eta^{\mathscr{T}_{n}} \frac{\sup _{i \in \mathscr{I}}\left\{\lambda_{\max }\left(Q_{i}\right)\right\}}{\inf _{i \in \mathscr{I}}\left\{\lambda_{\min }\left(Q_{i}\right)\right\}}, \quad \forall k \in \Gamma_{n}^{\mathcal{S}} .
\end{aligned}
$$

From (6), we have

$$
\begin{aligned}
\lambda_{1} R<P_{i}<\lambda_{2} R & \Longrightarrow \lambda_{1} I<R^{-1 / 2} P_{i} R^{-1 / 2}<\lambda_{2} I \\
& \Longrightarrow \lambda_{1} I<Q_{i}<\lambda_{2} I
\end{aligned}
$$

and from (17) we have

$$
\begin{aligned}
e^{T}(k) \operatorname{Re}(k) & <\left(\frac{\lambda_{2}}{\lambda_{1}}\right)^{f_{n} \mathscr{T}_{n}} \delta^{2} \eta^{\mathscr{T}_{n}} \frac{\sup _{i \in \mathscr{I}}\left\{\lambda_{\max }\left(Q_{i}\right)\right\}}{\inf _{i \in \mathscr{I}}\left\{\lambda_{\min }\left(Q_{i}\right)\right\}} \\
& <\left(\frac{\lambda_{2}}{\lambda_{1}}\right)^{1+f_{n} \mathscr{T}_{n}} \delta^{2} \eta^{\mathscr{T}_{n}} .
\end{aligned}
$$

From (8), we can obtain $e^{T}(k) \operatorname{Re}(k)<\varepsilon^{2}$. Therefore error dynamics (3) is finite-time stable.

Remark 11. The idea of Theorem 10 by using switching frequency $f_{n}$ is similar to the familiar conception, called average dwell time, which figures the average value of interval between consecutive switching instants [12]. From [12], we can define the average dwell time $\tau_{a} \geq 1 / f_{n}$ in the infinite time interval $\left[0, \mathscr{T}_{n}\right]$. A well-known fact in both asymptotic and finite-time stability is that the average dwell time should be sufficiently large to guarantee that the switched system is asymptotically or finite-time stable. By (7), we see that the switching frequency $f_{n}$ should be small enough, which obviously leads to sufficiently large average dwell time, to ensure that the error system is finite-time stable.

Theorem 10 gives a method to obtain finite-time observer, but Problem 6 has not been fully solved since the optimal boundary $\varepsilon$ is not taken into account, and, moreover, condition (8) in Theorem 10 is not an LMI; thus, Theorem 10 needs to be modified for optimal observer design.

Once the state bound $\varepsilon$ is not ascertained, the observer with minimal value $\varepsilon_{\min }$ is usually of great interest. With a fixed $\eta$ and letting $\lambda_{1}=1, \lambda_{2}=\mathscr{K}$, (6) and (8) become

$$
\begin{gathered}
R<P_{i}<\kappa R, \\
\varepsilon^{2}>\kappa^{1+f_{n} \mathscr{T}_{n}} \delta^{2} \eta^{\mathscr{T}_{n}} .
\end{gathered}
$$

Then, the following optimization problem can be constructed:

$$
\begin{array}{ll}
\min & \kappa \\
\text { s.t. } & (7) \text { and }(20)
\end{array}
$$

with optimized observer gains $L_{i}=P_{i}^{-1} X_{i}$ and minimal

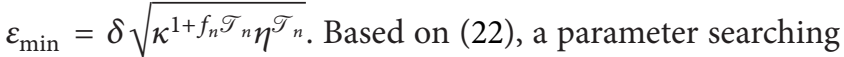
algorithm for designing optimal observer (3) can be formulated as in Algorithm 1.

Then, when the disturbance is considered, that is, $\omega(k) \neq$ 0 , we are going to design optimal finite-time observer, minimizing the bound $\varepsilon$, while the $\mathscr{H}_{\infty}$ performance is still maintained. The following theorem is given to solve Problem 9.

Theorem 12. Given a scalar $\gamma>0$ and considering switched system (1a) and (1b) and switching frequency $f_{n}$, if there exist a set of matrices $P_{i}, X_{i}$ and scalars $\lambda_{1}>0, \lambda_{2}>0$, and $\eta \geq 1$ such that

$$
\begin{gathered}
\lambda_{1} R<P_{i}<\lambda_{2} R \\
{\left[\begin{array}{ccc}
-\eta P_{i}+H_{i}^{T} H_{i} & H_{i}^{T} G_{i} & A_{i}^{T} P_{i}-C_{i}^{T} X_{i}^{T} \\
G_{i}^{T} H_{i} & G_{i}^{T} G_{i}-\frac{\gamma^{2}}{\eta^{T_{n}}} I & E_{i}^{T} P_{i}-F_{i}^{T} X_{i}^{T} \\
P_{i} A_{i}-X_{i} C_{i} & P_{i} E_{i}-X_{i} F_{i} & -P_{i}
\end{array}\right]<0,} \\
\left(\frac{\lambda_{2}}{\lambda_{1}}\right)^{f_{n} \mathscr{T}_{n}}\left(\eta^{\mathscr{T}_{n}} \lambda_{2} \delta^{2}+\gamma^{2} d^{2}\right)-\lambda_{1} \varepsilon^{2}<0,
\end{gathered}
$$

then error dynamics (3) with $L_{i}=P_{i}^{-1} X_{i}$ is finite-time bounded with $\mathscr{H}_{\infty}$ performance with respect to $\left(\delta, \varepsilon, d, \widehat{\gamma}, R, \mathscr{T}_{n}\right)$, where $\widehat{\gamma}=\gamma \sqrt{\left(\lambda_{2} / \lambda_{1}\right)^{f_{n} \mathscr{T}_{n}}}$.

Proof. Letting $V_{i}(k)=e^{T}(k) P_{i} e(k), i \in \mathscr{I}$ for each subsystem of error dynamics (3), we consider

$$
W_{i}(k)=z^{T}(k) z(k)-\frac{\gamma^{2}}{\eta^{\mathscr{T}_{n}}} \omega^{T}(k) \omega(k)+\Delta V_{i}(k)
$$

By letting $\xi^{T}(k)=\left[\begin{array}{ll}e^{T}(t) & \omega^{T}(t)\end{array}\right]$ and by simple manipulations, we see that

$$
\begin{aligned}
W_{i}(k)= & \xi^{T}(k) \\
& \times\left[\begin{array}{cc}
\widetilde{A}_{i}^{T} P_{i} \widetilde{A}_{i}-P_{i}+H_{i}^{T} H_{i} & \widetilde{A}_{i}^{T} P_{i} \widetilde{B}_{i}+H_{i}^{T} G_{i} \\
* & \widetilde{B}_{i}^{T} P_{i} \widetilde{B}_{i}+G_{i}^{T} G_{i}-\frac{\gamma^{2}}{\eta^{T_{n}}} I
\end{array}\right] \\
& \times \xi(k),
\end{aligned}
$$


Step 1. Initialize $\eta=1$, set a variation value $\Delta \eta>0$ and termination value $\bar{\gamma}$.

Step 2. Setting $\eta=\eta+\Delta \eta$, solve optimization problem (22) with fixed $\eta$.

Step 3. When the optimization problem (22) is solvable for the first time, the value of $\eta$ recorded as $\eta_{\min }$.

Then, if $\eta \geq \eta_{\min }+\bar{\eta}$, terminate the procedure, otherwise record parameters $\left(\eta, \varepsilon_{\min }\right)$ pair-wisely and go back to Step 2.

Step 4. Select the $\widetilde{\eta}$ with smallest $\varepsilon_{\min }$ recorded in Step 3. Obtain the locally optimized observer gains and ascertain the local optimal value of $\varepsilon_{\min }=\delta \sqrt{\kappa^{1+f_{n} \mathscr{T}_{n}} \eta^{\mathscr{T}_{n}}}$ near $\tilde{\eta}$ by an unconstrained nonlinear optimization approach.

Algorithm 1: Optimal finite-time observer design with minimal bound $\varepsilon_{\min }$.

where $\widetilde{A}_{i}=A_{i}-L_{i} C_{i}$ and $\widetilde{B}_{i}=E_{i}-L_{i} F_{i}$. From (24) and Schur complement formula, it follows that

$$
V_{i}(k+1)<\eta V_{i}(k)-z^{T}(k) z(k)+\frac{\gamma^{2}}{\eta^{T_{n}}} \omega^{T}(k) \omega(k) .
$$

At first, we consider the finite-time boundedness. Since $z^{T}(k) z(k) \geq 0,(28)$ always indicates

$$
V_{i}(k+1)<\eta V_{i}(k)+\frac{\gamma^{2}}{\eta^{\mathscr{T}_{n}}} \omega^{T}(k) \omega(k) .
$$

Iterating the above inequality, one has

$$
V_{i}(k)<\eta^{k-k_{m}^{n}} V_{i}\left(k_{m}^{n}\right)+\sum_{l=k_{m}^{n}}^{k-1} \eta^{k-l-1} \frac{\gamma^{2}}{\eta^{\mathscr{T}_{n}}} \omega^{T}(l) \omega(l) .
$$

Then, we let

$$
V(k)=\sum_{i \in \mathscr{I}} \theta_{i}(k) V_{i}(k)=\sum_{i \in \mathscr{I}} e^{T}(k) \theta_{i}(k) P_{i} e(k),
$$

where $\theta_{i}(\cdot): \mathbb{Z}^{+} \rightarrow\{0,1\}$ and $\sum_{i \in \mathcal{I}^{\mathcal{G}}} \theta_{i}(k)=1$, and from (23) indicating $V_{j}\left(k_{m}^{n}\right) / V_{i}\left(k_{m}^{n}\right)<\lambda_{2} / \lambda_{1}, i \neq j$, for all $(i, j) \in$ $\mathscr{I} \times \mathscr{I}$, the following results can be obtained:

$$
\begin{aligned}
V(k)< & \eta^{k-k_{0}^{n}}\left(\frac{\lambda_{2}}{\lambda_{1}}\right)^{N\left(k_{0}, k\right)} V\left(k_{0}^{n}\right) \\
& +\sum_{l=k_{0}^{n}}^{k-1} \eta^{k-l-1}\left(\frac{\lambda_{2}}{\lambda_{1}}\right)^{N(s, k)} \frac{\gamma^{2}}{\eta^{T_{n}}} \omega^{T}(l) \omega(l),
\end{aligned}
$$

where $N\left(k_{1}, k_{2}\right)$ implies the switching number in $\left[k_{1}, k_{2}\right)$. Then by $\sum_{l=k_{0}^{n}}^{M} \omega^{T}(l) \omega(l)<d^{2}, \lambda_{2} / \lambda_{1}>1$, it has

$$
\begin{aligned}
V(k)< & \eta^{k-k_{0}^{n}}\left(\frac{\lambda_{2}}{\lambda_{1}}\right)^{f_{n} \mathscr{T}_{n}} V\left(k_{0}^{n}\right) \\
& +\eta^{\mathscr{T}_{n}}\left(\frac{\lambda_{2}}{\lambda_{1}}\right)^{f_{n} \mathscr{T}_{n}} \frac{\gamma^{2}}{\eta^{\mathscr{T}_{n}}} \sum_{l=k_{0}^{n}}^{k-1} \omega^{T}(l) \omega(l) \\
< & \eta^{\mathscr{T}_{n}}\left(\frac{\lambda_{2}}{\lambda_{1}}\right)^{f_{n} \mathscr{T}_{n}} V\left(k_{0}^{n}\right)+\left(\frac{\lambda_{2}}{\lambda_{1}}\right)^{f_{n} \mathscr{T}_{n}} \gamma^{2} d^{2} .
\end{aligned}
$$

Following similar guidelines in Theorem 10, we see

$$
\begin{gathered}
V(k) \geq \inf _{i \in \mathscr{I}}\left\{\lambda_{\min }\left(Q_{i}\right)\right\} e^{T}(k) \operatorname{Re}(k), \\
V\left(k_{0}^{n}\right) \leq \sup _{i \in \mathscr{I}}\left\{\lambda_{\max }\left(Q_{i}\right)\right\} \delta^{2},
\end{gathered}
$$

where $Q_{i}=R^{-1 / 2} P_{i} R^{-1 / 2}, i \in \mathscr{I}$. Altogether (33) (35), the following inequality can be derived as

$e^{T}(k) \operatorname{Re}(k)$

$<\frac{\eta^{\mathscr{T}_{n}}\left(\lambda_{2} / \lambda_{1}\right)^{f_{n} \mathscr{T}_{n}} \sup _{i \in \mathcal{F}}\left\{\lambda_{\max }\left(Q_{i}\right)\right\} \delta^{2}+\left(\lambda_{2} / \lambda_{1}\right)^{f_{n} \mathscr{T}_{n}} \gamma^{2} d^{2}}{\inf _{i \in \mathscr{F}}\left\{\lambda_{\min }\left(Q_{i}\right)\right\}}$,

$\forall k \in \Gamma_{n}^{s}$

From (23), we have

$$
\begin{aligned}
\lambda_{1} R<P_{i}<\lambda_{2} R & \Longrightarrow \lambda_{1} I<R^{-1 / 2} P_{i} R^{-1 / 2}<\lambda_{2} I \\
& \Longrightarrow \lambda_{1} I<Q_{i}<\lambda_{2} I .
\end{aligned}
$$

Thus, (36) becomes

$$
e^{T}(k) \operatorname{Re}(k)<\frac{\eta^{\mathscr{T}_{n}}\left(\lambda_{2} / \lambda_{1}\right)^{f_{n} \mathscr{T}_{n}} \lambda_{2} \delta^{2}+\left(\lambda_{2} / \lambda_{1}\right)^{f_{n} \mathscr{T}_{n}} \gamma^{2} d^{2}}{\lambda_{1}} .
$$

By (25), the finite-time boundness is obtained; that is, $e^{T}(k) \operatorname{Re}(k)<\varepsilon^{2}$.

Then, the $\mathscr{H}_{\infty}$ performance is considered. Iterating (28), we get

$$
\begin{aligned}
V_{i}(k) \leq & \eta^{k-k_{m}^{n}} V_{i}\left(k_{m}^{n}\right) \\
& +\sum_{l=k_{m}^{n}}^{k-1} \eta^{k-l-1}\left[\frac{\gamma^{2}}{\eta^{T_{n}}} \omega^{T}(l) \omega(l)-z^{T}(l) z(l)\right] .
\end{aligned}
$$


And similarly, it yields

$$
\begin{aligned}
V(k)< & \eta^{k-k_{0}^{n}}\left(\frac{\lambda_{2}}{\lambda_{1}}\right)^{N\left(k_{0}, k\right)} V\left(k_{0}^{n}\right) \\
+ & \sum_{l=k_{0}^{n}}^{k-1} \eta^{k-l-1}\left(\frac{\lambda_{2}}{\lambda_{1}}\right)^{N(s, k)} \\
& \times\left[\frac{\gamma^{2}}{\eta^{\mathscr{T}_{n}}} \omega^{T}(l) \omega(l)-z^{T}(l) z(l)\right] .
\end{aligned}
$$

Under zero-initial condition, that is, $V\left(k_{0}^{n}\right)=0$ and by $V(k) \geq 0$, it implies

$$
\begin{aligned}
\sum_{l=k_{0}^{n}}^{k-1} \eta^{k-l-1}\left(\frac{\lambda_{2}}{\lambda_{1}}\right)^{N(s, k)}\left[\frac{\gamma^{2}}{\eta^{M}} \omega^{T}(l) \omega(l)-z^{T}(l) z(l)\right] \geq 0 \\
\quad \Longrightarrow \sum_{l=k_{0}^{n}}^{k-1} \eta^{k-l-1}\left(\frac{\lambda_{2}}{\lambda_{1}}\right)^{N(s, k)} z^{T}(l) z(l) \\
\leq \frac{\gamma^{2}}{\eta^{M}} \sum_{l=k_{0}^{n}}^{k-1} \eta^{k-l-1}\left(\frac{\lambda_{2}}{\lambda_{1}}\right)^{N(s, k)} \omega^{T}(l) \omega(l) \\
\Longrightarrow \sum_{l=k_{0}^{n}}^{k-1} z^{T}(l) z(l) \\
\leq \frac{\gamma^{2}}{\eta^{M}} \sum_{l=k_{0}^{n}}^{k-1} \eta^{\mathscr{T}_{n}}\left(\frac{\lambda_{2}}{\lambda_{1}}\right)^{f_{n} \mathscr{T}_{n}} \omega^{T}(l) \omega(l) \\
\Longrightarrow \sum_{l=k_{0}^{n}}^{k-1} z^{T}(l) z(l) \leq\left(\frac{\lambda_{2}}{\lambda_{1}}\right)^{f_{n} \mathscr{T}_{n}} \gamma^{2} \sum_{l=k_{0}^{n}}^{k-1} \omega^{T}(l) \omega(l)
\end{aligned}
$$

Therefore, the $\mathscr{H}_{\infty}$ performance is established. We can conclude that the error dynamics (3) is finite-time bounded with $\mathscr{H}_{\infty}$ performance with respect to $\left(\delta, \varepsilon, d, \widehat{\gamma}, R, \mathscr{T}_{n}\right)$, where $\widehat{\gamma}=\gamma \sqrt{\left(\lambda_{2} / \lambda_{1}\right)^{f_{n} \mathscr{T}_{n}}}$.

Remark 13. In Theorem 12, we find that the $\mathscr{H}_{\infty}$ performance index $\hat{\gamma}=\gamma \sqrt{\left(\lambda_{2} / \lambda_{1}\right)^{f_{n} \mathscr{T}_{n}}}$ which relates to the switching frequency $f_{n}$; if the $f_{n}$ is large, which means switching behaviour frequently occurs during the interval, $\widehat{\gamma}$ also becomes larger implying that the $\mathscr{H}_{\infty}$ performance will be worsened. Particularly, if there exists no switching, that is, $f_{n}=0$, it becomes the $\gamma$ which is not influenced by switching.

Remark 14. Comparing Theorem 12 with Theorem 10, Theorem 12 can be viewed as an extension of Theorem 10, or Theorem 10 is a particular case with $\omega(k) \equiv 0$ in Theorem 12 . For example, letting $E_{i}=F_{i}=G_{i}=H_{i}=0$ in (24), it becomes (7) in Theorem 10, and letting $d=0$ implying $\omega(k) \equiv 0,(25)$ in Theorem 12 is exactly the same as (8) in Theorem 10.
Similar to the solution to Problem 6, we have to modify Theorem 12 to solve the optimal observer design Problem 9. With a fixed $\eta$ and by letting $\lambda_{1}=1, \lambda_{2}=\kappa$, (23) becomes

$$
R<P_{i}<\kappa R
$$

And (25) becomes

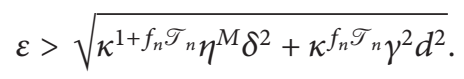

Then, the following optimization problem can be constructed:

$$
\begin{aligned}
& \min \kappa \\
& \text { s.t. } \quad(24) \text { and }(42) \text {, }
\end{aligned}
$$

with optimized observer gains $L_{i}=P_{i}^{-1} X_{i}$ and minimal

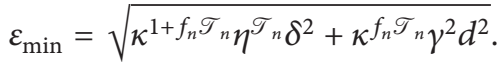

Then based on (44), a similar algorithm for designing $\mathscr{H}_{\infty}$ optimal observer (2) can be formulated as in Algorithm 2.

In both Algorithms 1 and 2, if we can find a feasible solution with parameter $\eta=1$, by the discussion above, we know that the designed observer can guarantee both finitetime and asymptotic stability of error dynamics. But in a general situation, we often obtain observers with $\eta>1$, and only finite-time stability can be established. Thus, additional asymptotic observers for each subsystem have to be designed to ensure asymptotic stability, which can be easily obtained by linear system theory.

\section{Further Discussions on Improved Results}

Theorems 10 and 12 in the previous section are derived based on the switching frequency $f_{n}$; furthermore, if more information about the switching signal is known, that is, the total activation time of each subsystem is available, we can derive another less conservative result on observer design. In Theorems 10 and 12, the subobservers might be designed with only one parameter $\eta \geq 1$, which includes the two cases that the asymptotic stability of error dynamics is also established when $\eta=1$ or not when $\eta>1$. When the total activation time of each subsystem can be prespecified, multiple parameters $\eta_{i}$ are introduced in observer design. $\mathscr{T}_{n}^{i}$ is used to denote the total activation time of subsystem $i$ in short-time switching interval $\Gamma_{n}^{s}$. It is explicit that $\sum_{i \in \mathscr{I}} \mathscr{T}_{n}^{i}=$ $\mathscr{T}_{n}$. Then some improved results with less conservativeness can be derived. Because Theorem 12 covers Theorem 10, as Remark 13 indicates, we consider the general case of Theorem 12, where the $\mathscr{H}_{\infty}$ performance is concerned.

Theorem 15. Given a scalar $\gamma>0$ and considering switched system (1a) and (1b) and switching frequency $f_{n}$, if there exist 
Step 1. Initialize $\eta=1$, set a variation value $\Delta \eta>0$ and termination value $\bar{\gamma}$.

Step 2. Setting $\eta=\eta+\Delta \eta$, solve optimization problem (44) with fixed $\eta$.

Step 3. When the optimization problem (44) is solvable for the first time, the value of $\eta$ recorded as $\eta_{\min }$.

Then, if $\eta \geq \eta_{\min }+\bar{\eta}$, terminate the procedure, otherwise record parameters $\left(\eta, \varepsilon_{\min }\right)$ pair-wisely and go back to Step 2 .

Step 4. Select the $\tilde{\eta}$ with smallest $\varepsilon_{\min }$ recorded in Step 3. Obtain the locally optimized observer gains and ascertain the local optimal value of $\varepsilon_{\min }=\sqrt{\kappa^{1+f_{n} \mathscr{T}_{n}} \eta^{\mathscr{T}_{n}} \delta^{2}+\kappa^{f_{n} \mathscr{T}_{n}} \gamma^{2} d^{2}}$ near $\tilde{\eta}$ by an unconstrained nonlinear optimization approach.

Algorithm 2: Optimal $\mathscr{H}_{\infty}$ finite-time observer design with minimal bound $\varepsilon_{\min }$.

a set of matrices $P_{i}, X_{i}$ and scalars $\lambda_{1}>0, \lambda_{2}>0$, and $\eta_{i} \geq 1$ such that

$$
\begin{gathered}
\lambda_{1} R<P_{i}<\lambda_{2} R \\
{\left[\begin{array}{ccc}
-\eta_{i} P_{i}+H_{i}^{T} H_{i} & H_{i}^{T} G_{i} & A_{i}^{T} P_{i}-C_{i}^{T} X_{i}^{T} \\
G_{i}^{T} H_{i} & G_{i}^{T} G_{i}-\frac{\gamma^{2}}{\prod_{i \in \mathcal{G}} \eta_{i}^{\mathcal{T}^{i}}} I & E_{i}^{T} P_{i}-F_{i}^{T} X_{i}^{T} \\
P_{i} A_{i}-X_{i} C_{i} & P_{i} E_{i}-X_{i} F_{i} & -P_{i}
\end{array}\right]<0} \\
\left(\frac{\lambda_{2}}{\lambda_{1}}\right)^{f_{n} \mathscr{T}_{n}}\left(\prod_{i \in \mathscr{I}} \eta_{i}^{\mathscr{T}^{i}}{ }^{n} \lambda_{2} \delta^{2}+\gamma^{2} d^{2}\right)-\lambda_{1} \varepsilon^{2}<0
\end{gathered}
$$

then error dynamics (3) with $L_{i}=P_{i}^{-1} X_{i}$ is finite-time bounded with $\mathscr{H}_{\infty}$ performance with respect to $\left(\delta, \mathcal{\varepsilon}, d, \widehat{\gamma}, R, \mathscr{T}_{n}\right)$, where $\widehat{\gamma}=\gamma \sqrt{\left(\lambda_{2} / \lambda_{1}\right)^{f_{n} \mathscr{T}_{n}}}$

Proof. By $\sum_{i \in \mathscr{I}} \mathscr{T}_{n}^{i}=\mathscr{T}_{n}$ and letting $\eta=\max _{i \in \mathscr{F}}\left\{\eta_{i}\right\}$, it is easy to get $\prod_{i \in \mathcal{F}} \eta_{i}^{\mathscr{T}^{i}} \leq \eta^{\mathscr{T}_{n}}$; therefore, (45) (47) directly leads to (23) (25) being satisfied. Based on Theorem 10, the proof is completed.

Remark 16. In the proof line, we see that Theorem 12 is the case that we choose $\eta=\max _{i \in \mathscr{I}}\left\{\eta_{i}\right\}$ in Theorem 15; thus, we can easily see that $\eta^{\mathscr{T}_{n}} \geq \prod_{i \in \mathcal{F}} \eta_{i}^{\mathscr{T}^{i}}$ which indicates the less conservativeness of Theorem 15 compared with Theorem 12. But, since more parameters searching is required in Theorem 15, more computation cost such as a genetic algorithm is needed for using Theorem 15 . Conservativeness and computation cost comparisons between the two theorems will be given by a numerical example later.

Similarly, a less conservative result for Theorem 10 can be obtained as a corollary of Theorem 15 .

Corollary 17. Consider switched system (1a) and (1b) with $\omega(k) \equiv 0$ and switching frequency $f_{n}$. If there exist a set of matrices $P_{i}, X_{i}$ and scalars $\lambda_{1}>0, \lambda_{2}>0$, and $\eta_{i} \geq 1$ such that

$$
\begin{gathered}
\lambda_{1} R<P_{i}<\lambda_{2} R, \\
{\left[\begin{array}{cc}
-\eta_{i} P_{i} & A_{i}^{T} P_{i}-C_{i}^{T} X_{i}^{T} \\
P_{i} A_{i}-X_{i} C_{i} & -P_{i}
\end{array}\right]<0,} \\
\left(\frac{\lambda_{2}}{\lambda_{1}}\right)^{1+f_{n} \mathscr{T}_{n}} \delta^{2} \prod_{i \in \mathscr{I}} \eta_{i}^{\mathscr{T}^{i}}-\varepsilon^{2}<0,
\end{gathered}
$$

then error dynamics (3) with $L_{i}=P_{i}^{-1} X_{i}$ is finite-time stable with respect to $\left(\delta, \varepsilon, R, \mathscr{T}_{n}\right)$.

To design optimal observer with minimal value $\varepsilon_{\text {min }}$, a similar optimization problem can be constructed; however, the situation by letting $\lambda_{1}=1, \lambda_{2}=\kappa$ is more complex due to (47) or (50); that is,

$$
\begin{gathered}
\varepsilon>\sqrt{\kappa^{1+f_{n} \mathscr{T}_{n}}\left(\prod_{i \in \mathscr{I}} \eta_{i}^{\mathscr{T}^{i}} \delta^{2}+\kappa^{f_{n} \mathscr{T}_{n}} \gamma^{2} d^{2}\right)}, \\
\varepsilon^{2}>\kappa^{1+f_{n} \mathscr{T}_{n}} \delta^{2} \prod_{i \in \mathscr{I}} \eta_{i}^{\mathscr{T}^{i}} .
\end{gathered}
$$

Remark 18. According to (51), the state bound $\varepsilon$ is affected by many factors including several parameters such as $\kappa$ and multiple $\eta_{i}$ for subsystems. Hence, to obtain the optimal observer gains with minimal value $\varepsilon_{\text {min }}$ is a complex nonlinear optimization problem based on Theorem 15. As a powerful tool solving complex nonlinear optimization problem, the genetic algorithm, which is an optimization method inspired by the principles of Darwinian evolution, is introduced. As for the details about genetic algorithm, the reader is referred to [28] and many other textbooks and literature. 


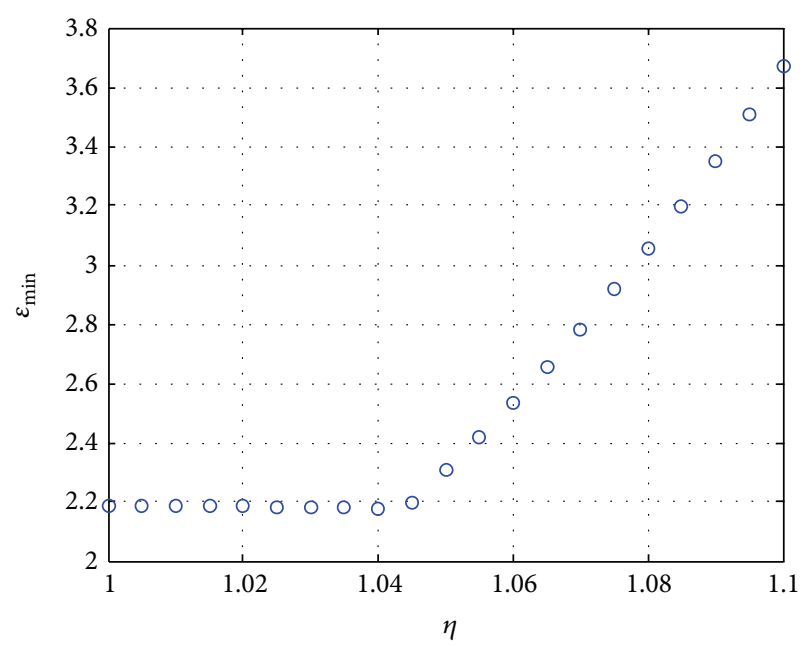

FIGURE 1: The value of $\varepsilon_{\min }$ with different $\eta$.

\section{Numerical Example}

Consider a switched discrete-time linear system with two subsystems as follows:

$$
\begin{aligned}
& A_{1}=\left[\begin{array}{cc}
-1.2566 & -0.9414 \\
-0.3472 & -1.1746
\end{array}\right], \\
& A_{2}=\left[\begin{array}{cc}
0.1737 & 1.0641 \\
-0.1161 & -0.2454
\end{array}\right], \\
& C_{1}^{T}=\left[\begin{array}{l}
0.0714 \\
0.3165
\end{array}\right], \\
& C_{2}^{T}=\left[\begin{array}{l}
0.4998 \\
1.2781
\end{array}\right] .
\end{aligned}
$$

The switching signal is considered as a periodical switching signal in interval $[0,20]$, the switching sequence is given as $\mathcal{S}_{1}:=\{0,4,8,12,16,20\}$, and the initial subsystem is subsystem 1; thus, the average dwell time $f_{n}=0.25, \mathscr{T}_{1}^{1}=12$, and $\mathscr{T}_{1}^{2}=8$. The state estimation design objective in shorttime switching interval $[0,20]$ is to design a set of observer gains minimizing the value of $\|e(k)\|$, for all $k \in \Gamma_{1}^{\mathcal{s}}$, when the initial value of error state satisfies $\|e(0)\| \leq \sqrt{2}$. Thus we can choose parameters $\delta=\sqrt{2}, R=I$, and $\mathscr{T}_{n}=20$. To compare the conservativeness of two approaches based on Theorem 10 and Corollary 17, we design observer through them, respectively.

At first, we apply Algorithm 1 based on Theorem 10.

Step 1. Initialize parameters $\eta=1, \Delta \eta=0.005$, and $\bar{\eta}=1.1$.

Step 2. Solving optimization problem (22), find $\varepsilon_{\min }$ with different $\eta$ as shown in Figure 1 .

It is shown that the minimal value of $\varepsilon_{\min }$ can be obtained when $\widetilde{\eta}=1.04$.

Step 3. Ascertain the local optimal value of $\varepsilon_{\min }^{*}=2.1769$ with $\eta=1.0411$ near $\tilde{\eta}=1.04$ by an unconstrained nonlinear

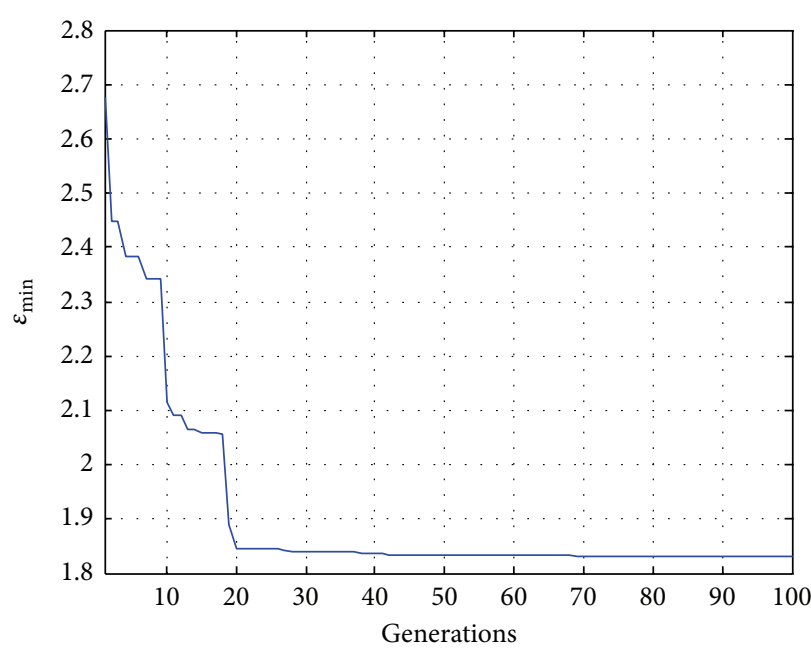

FIGURE 2: The value of $\varepsilon_{\min }$ along with 100 generations.

optimization approach with the following optimal observer gains:

$$
\begin{aligned}
& L_{1}=\left[\begin{array}{ll}
-3.6817 & -3.7671
\end{array}\right]^{T}, \\
& L_{2}=\left[\begin{array}{ll}
0.7691 & -0.1973
\end{array}\right]^{T} .
\end{aligned}
$$

Step 4. Then, we apply the genetic algorithm with 100 generations based on Corollary 17 ; the best value of $\varepsilon_{\min }$ along with the generations is given in Figure 2.

The optimal value of $\varepsilon_{\text {min }}^{*}=1.8320$ with $\eta_{1}=1.0410$ and $\eta_{2}=1.0000$, and optimal observer gains are

$$
\begin{aligned}
& L_{1}=\left[\begin{array}{ll}
-3.6836 & -3.7608
\end{array}\right]^{T}, \\
& L_{2}=\left[\begin{array}{ll}
0.7760 & -0.1967
\end{array}\right]^{T} .
\end{aligned}
$$

Comparing the two results, since genetic algorithm is applied by Corollary 17, the computation cost increases as generations increase. The computation cost of Theorem 10 is much less than that of Corollary 17, which only equals to one generation step in Corollary 17. But, on the other hand, the advantages of Corollary 17 are very obvious; the optimal value of minimal bound $\varepsilon_{\text {min }}^{*}$ derived by Corollary 17 after 100 generation evolution is smaller than that derived by Theorem 10. Hence, we can see that the result by Corollary 17 is less conservative than that by Theorem 10 in this numerical example.

Furthermore, since $\eta_{1}>1$, the asymptotic observers for long intervals without switching should be designed by traditional asymptotic observer design approach.

\section{Conclusions}

In this paper, the state estimation problem for switched system during a finite-time interval is addressed in the framework of switching frequency. Based on the conception of finite-time stability, an optimal observer is designed to 
minimize the bound of error state, and then the results are extended to the case concerned with $\mathscr{H}_{\infty}$ performance, where the bound of error state is minimized while the $\mathscr{H}_{\infty}$ performance in the finite-time interval is maintained. Particularly, when the total activation time is known, a less conservative result can be derived and an optimization problem can be solved with the help of the genetic algorithm. Since many actual switched systems exhibit short-time switching property and hereby can be modeled by short-time switched systems, our theoretical results are supposed to be widely used in real-world switched systems potentially such as extension from synchronous switching case to asynchronous switching case, which should be further considered in future work.

\section{Conflict of Interests}

The authors declare that there is no conflict of interests regarding the publication of this paper.

\section{References}

[1] D. Liberzon, Switching in Systems and Control, Birkhauser, Boston, Mass, USA, 2003.

[2] Z. Sun and S. S. Ge, Switched Linear Systems-Control and Design, Springer, London, UK, 2005.

[3] R. A. Decarlo, M. S. Branicky, S. Pettersson, and B. Lennartson, "Perspectives and results on the stability and stabilizability of hybrid systems," Proceedings of the IEEE, vol. 88, no. 7, pp. 10691082, 2000.

[4] A. Balluchi, M. D. Benedetto, C. Pinello, C. Rossi, and A. Sangiovanni-Vincentelli, "Cut-off in engine control: a hybrid system approach," in Proceedings of the 36th IEEE Conference on Decision and Control, pp. 4720-4725, 1997.

[5] W. Zhang, M. S. Branicky, and S. M. Phillips, "Stability of networked control systems," IEEE Control Systems Magazine, vol. 21, no. 1, pp. 84-97, 2001.

[6] K. S. Narendra, O. A. Driollet, M. Feiler, and K. George, "Adaptive control using multiple models, switching and tuning," International Journal of Adaptive Control and Signal Processing, vol. 17, no. 2, pp. 87-102, 2003.

[7] C. Sreekumar and V. Agarwal, "A hybrid control algorithm for voltage regulation in DC-DC boost converter," IEEE Transactions on Industrial Electronics, vol. 55, no. 6, pp. 2530-2538, 2008.

[8] X. Sun, G. Liu, W. Wang, and D. Rees, " $L_{2}$-gain of systems with input delays and controller temporary failure: zero-order hold model," IEEE Transactions on Control Systems Technology, vol. 19, no. 3, pp. 699-706, 2011.

[9] K. S. Narendra and J. Balakrishnan, "A common Lyapunov function for stable LTI systems with commuting A-matrices," IEEE Transactions on Automatic Control, vol. 39, no. 12, pp. 2469-2471, 1994.

[10] M. S. Branicky, "Multiple Lyapunov functions and other analysis tools for switched and hybrid systems," IEEE Transactions on Automatic Control, vol. 43, no. 4, pp. 475-482, 1998.

[11] J. Daafouz, P. Riedinger, and C. Iung, "Stability analysis and control synthesis for switched systems: a switched Lyapunov function approach," Institute of Electrical and Electronics Engineers. Transactions on Automatic Control, vol. 47, no. 11, pp. 1883-1887, 2002.
[12] J. P. Hespanha and A. S. Morse, "Stability of switched systems with average dwell-time," in Proceedings of the 38th IEEE Conference on Decision and Control (CDC '99), pp. 2655-2660, December 1999.

[13] Y. E. Wang, J. Zhao, and B. Jiang, "Stabilization of a class of switched linear neutral systems under asynchronous switching," IEEE Transactions on Automatic Control, vol. 58, no. 8, pp. 21142119, 2013.

[14] H. Lin and P. J. Antsaklis, "Stability and stabilizability of switched linear systems: a survey of recent results," IEEE Transactions on Automatic Control, vol. 54, no. 2, pp. 308-322, 2009.

[15] D. Luenberger, "Observers for multivariable systems," IEEE Transaction on Automatic Control, vol. 11, no. 2, pp. 190-197, 1966.

[16] A. Alessandri and P. Coletta, "Switching observers for continuous time and discrete-time linear systems," in Proceedings of the American Control Conference, pp. 2516-2521, Arlington, Va, USA, 2001.

[17] A. L. Juloski, W. P. M. H. Heemels, and S. Weiland, "Observer design for a class of piece-wise affine systems," in Proceeding of the 41st IEEE Conference on Decision and Control, vol. 3, pp. 2606-2611, December 2002.

[18] A. L. J. Juloski, W. P. M. H. Heemels, Y. Boers, and F. Verschure, "Two Approaches to State Estimation for a Class of Piecewise Affine Systems," in Proceedings of the 42nd IEEE Conference on Decision and Control, pp. 143-148, December 2003.

[19] S. Pettersson, "Observer design for switched systems using multiple quadratic lyapunov functions," in Proceedings of the 20th IEEE International Symposium on Intelligent Control (ISIC '05), pp. 262-267, Limassol, Cyprus, June 2005.

[20] W. Chen and S. Mehrdad, "Observer design for linear switched control systems," in Proceedings of the American Control Conference (AAC '04), pp. 5796-5801, July 2004.

[21] F. Amato and M. Ariola, "Finite-time control of discrete-time linear systems," IEEE Transactions on Automatic Control, vol. 50, no. 5, pp. 724-729, 2005.

[22] F. Amato, R. Ambrosino, M. Ariola, and C. Cosentino, "Finitetime stability of linear time-varying systems with jumps," Automatica, vol. 45, no. 5, pp. 1354-1358, 2009.

[23] R. Ambrosino, F. Calabrese, C. Cosentino, and G. De Tommasi, "Sufficient conditions for finite-time stability of impulsive dynamical systems," IEEE Transactions on Automatic Control, vol. 54, no. 4, pp. 861-865, 2009.

[24] W. Xiang and J. Xiao, " $H$ finite-time control for switched nonlinear discrete-time systems with norm-bounded disturbance," Journal of the Franklin Institute, vol. 348, no. 2, pp. 331-352, 2011.

[25] W. Xiang and J. Xiao, "Finite-time stability and stabilisation for switched linear systems," International Journal of Systems Science, vol. 44, no. 2, pp. 384-400, 2013.

[26] W. Xiang, J. Xiao, and M. N. Iqbal, "Asymptotic stability, $l_{2}$ gain boundness analysis and control synthesis for switched systems: a switching frequency approach," vol. 26, no. 4, pp. 350-373, 2012.

[27] D. J. Leith, R. N. Shorten, W. E. Leithead, O. Mason, and P. Curran, "Issues in the design of switched linear control systems: a benchmark study," International Journal of Adaptive Control and Signal Processing, vol. 17, no. 2, pp. 103-118, 2003.

[28] D. E. Goldberg, Genetic Algorithms in Search, Optimization and Machine Learning, Addison Wesley, New York, NY, USA, 1989. 


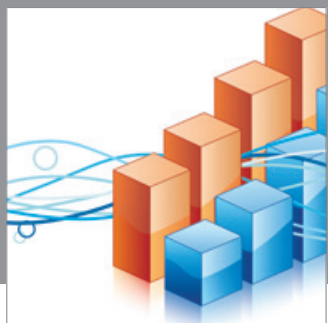

Advances in

Operations Research

mansans

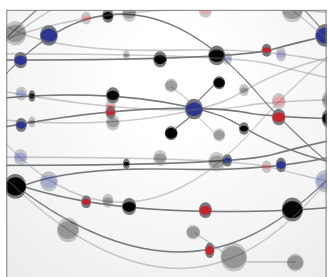

The Scientific World Journal
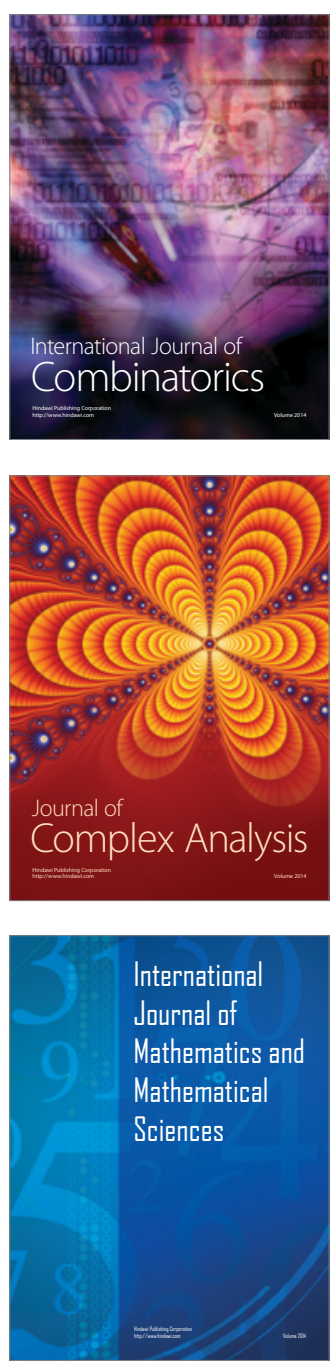
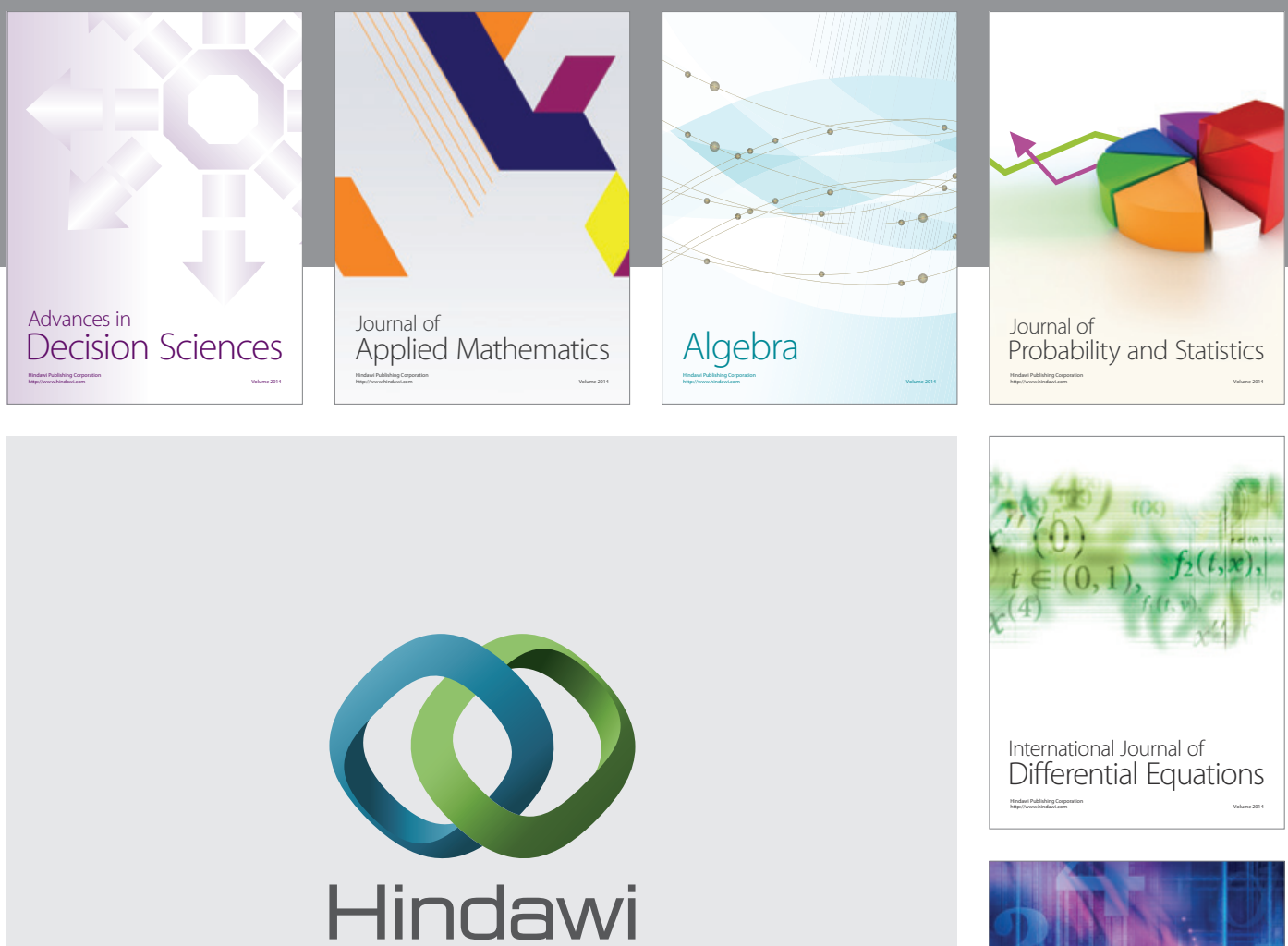

Submit your manuscripts at http://www.hindawi.com
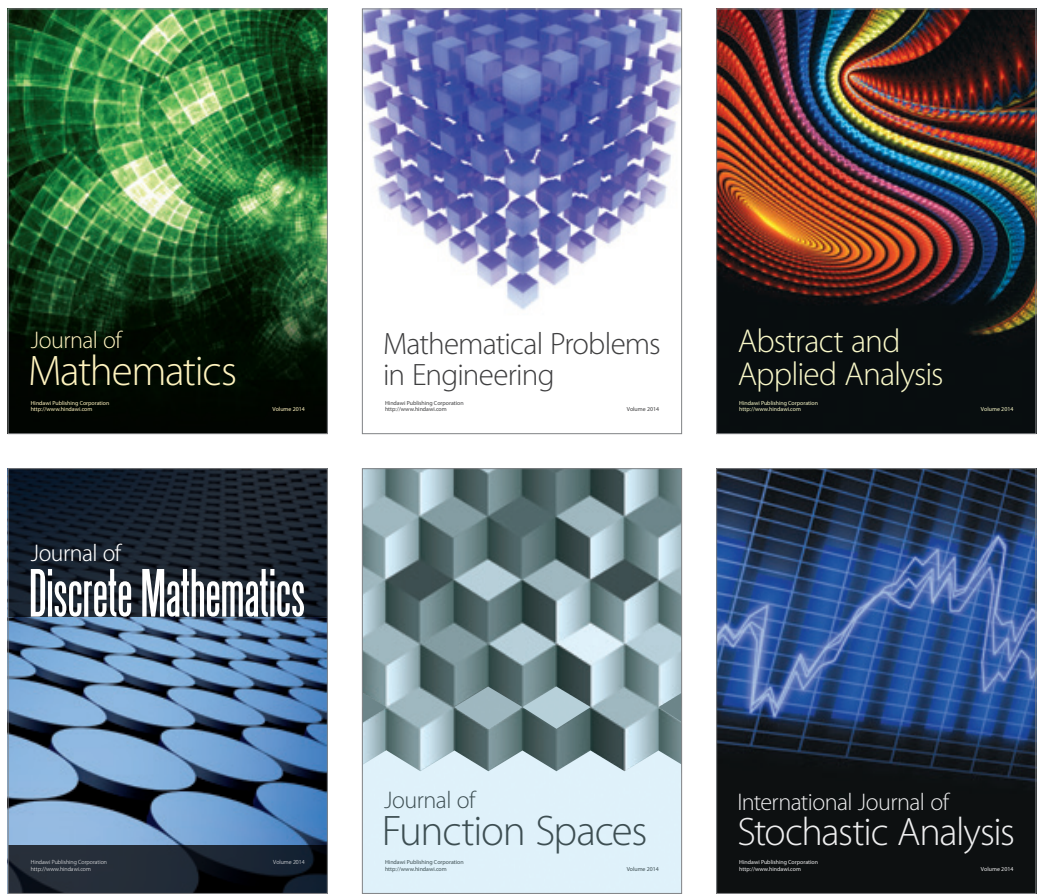

Journal of

Function Spaces

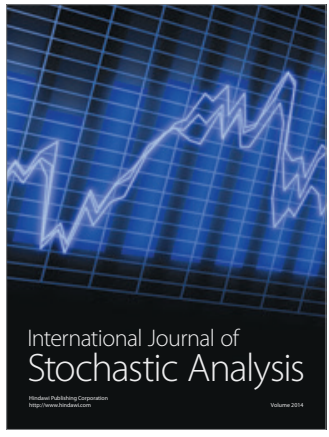

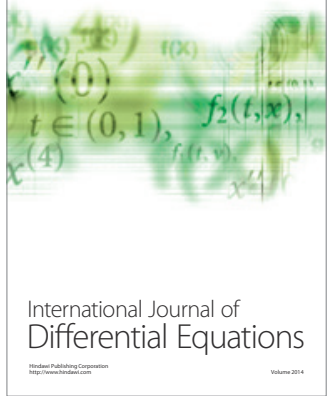
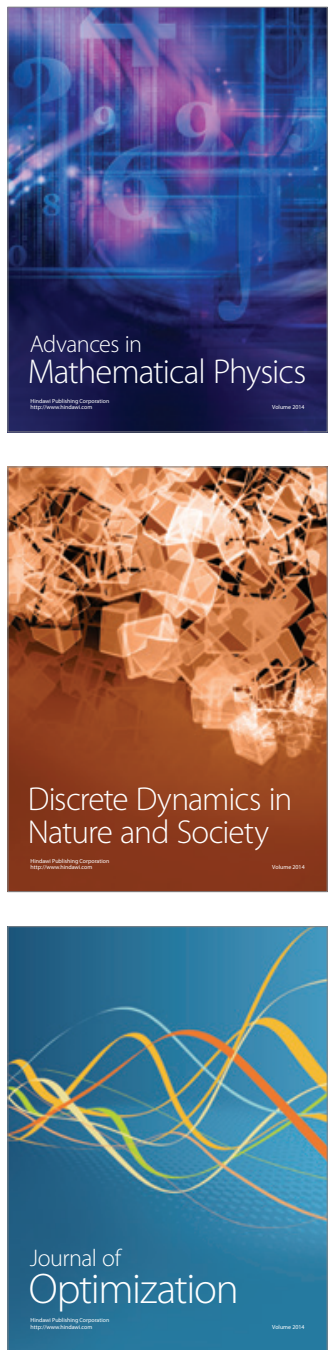